\title{
Remaining useful life prediction for ball bearings based on health indicators
}

\author{
Zeina Al Masry*, Patrick Schaible, Noureddine Zerhouni and Christophe Varnier \\ FEMTO-ST institute, Univ. Bourgogne Franche-Comté, CNRS, ENSMM, Besançon, 25000, France
}

\begin{abstract}
Uncertainty in remaining useful life (RUL) prediction is nowadays a scientific problem that occupies industrials. Many prognostic models have been developed to respond to this issue from probabilistic to non-probabilistic approaches. In this paper, we deal with a nonprobabilistic model for RUL prediction. For this purpose, we propose a model, which is based on health indicators information, that allows to estimate the RUL of ball bearings. The method is applied to simulated data provided by the PRONOSTIA platform designed and realized at AS2M department of FEMTO- ST Institute.
\end{abstract}

\section{Context of the work}

Nowadays, industries seek to improve their maintenance strategies in order to increase the lifetime of their equipments. Many of them are implementing Prognostics and Health Management (PHM) methodology, which seems to be a very promising strategy to minimize the overall cost and to improve the availability of a system. PHM has four main pillars: data processing, diagnostic, prognostic and decision-making (see $[1,2])$. We here are interested in the third pillar (prognostic) for ball bearings modeling, which aims to predict the remaining useful life (RUL) of the system. Recall that ball bearings plays a crucial role rotating machines. The failure of ball bearings may affect the functioning of the entire equipment. Thus, modeling the degradation process of the ball bearings allows to anticipate some failures. This paper aims to propose an adapted approach for ball bearings RUL prediction based on the data provided by PRONOSTIA platform (see [3]). In the literature, many techniques have been used for RUL estimation. Those techniques can be classified into model-based approach, data-driven approach and hybrid approach (see [4] for an overview on the prognostics approaches). In this paper, we considered a data-driven approach, which is based on the data provided by the sensors. Before presenting the approach, we first present the platform. PRONOSTIA is is an experimentation platform dedicated to test bearings fault detection and validate diagnostic and prognostic approaches. As the ball bearings are mostly responsable of the failures of rotating machines, it is therefore reasonable to build a testing environment to understand the process of the degradation of bearings. The platform PRONOSTIA has been developed at AS2M department of FEMTO-ST. It aims to provide real experimental data that characterize the degradation of ball bearings through two sensors: vibration and temperature. Figure 1 shows an overview of the platform, which is composed of 3 parts: rotating part, degradation part and a measurement part.

\footnotetext{
* Corresponding author: zeina.almasry@,femto-st.fr
} 


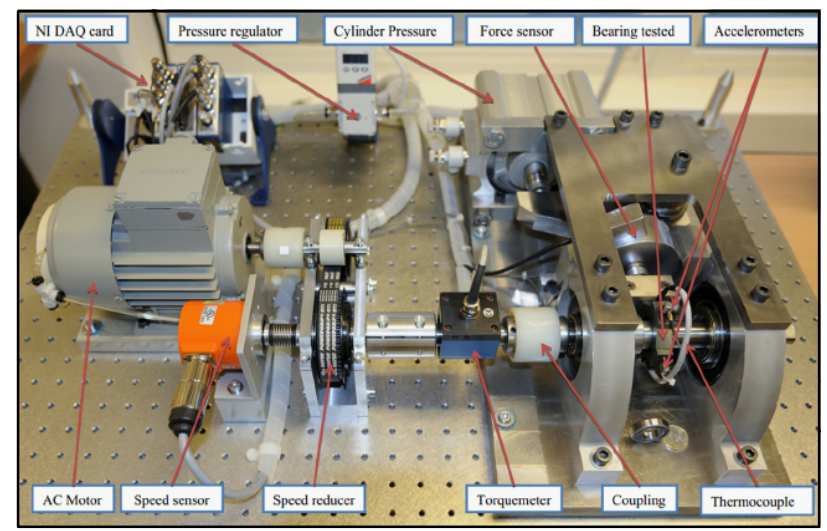

Fig. 1. PRONOSTIA platform

\section{Proposed approach and simulation results}

We now come to present the used techniques for ball bearing RUL's estimation. We followed the approach introduced in [5]. The proposed method is a component based method. It allows to estimate the RUL of the whole system, which is depending on the RUL of the critical parts. The critical components are identified by the user and the main parameters of the degradation. Different sensors are then used such as vibration, temperature or acoustic. As mentioned in Section 1, we here are interested in PRONOSTIA data platform. In this case, the critical component is the bearing that is tested in the PRONOSTIA environment and the sensors are already selected (vibration sensors, temperature, force sensor, torquemeter and speed sensor). This paper focuses on the vibration signals, which allows health indicator (HI) extraction. The HI matches the difference between the nominal and the degraded vibration signal and it is obtained by calculating the correlation of the vibration signals of the nominal and the defect bearing. This indicator is then used to determine the progression of the degradation and also to estimate the RUL.

The proposed method is applied on two signals of the PRONOSTIA with the same physical characteristic, a nominal bearing without degradation and a degraded bearing. The test duration for both bearings are the same with 4 hours and 33 minutes as well as the applied force of $5000 \mathrm{~N}$ and the speed about 1500 rotation per minute. Firstly, a resampling of the HI was made to reduce the sample size. Afterwards it got filtered by using a moving average filter with a window size of 8 . Secondly, a mathematical model is selected to describe the progression of the HI and to later on predict the RUL. For this case two methods were used: the first one is a polynomial model and the second one is an exponential model. Only the exponential model is presented in the following. The remaining useful lifetime is described by the time difference of the current time and the time which the regression model reaches the failure state and it is computed using the following equations:

$$
R U L(t)=T-H I^{-1}(t)
$$




$$
H I(t)=0,6085 e^{-4,6 * 10-5 t}
$$

where $H I^{-1}(t)$ is inverse function of the corresponding model and $\mathrm{T}$ the time when the failure occurs.

Figure 2 shows the result for the estimated RUL using exponential model and the real RUL. It is clear that it fits well the real RUL of the system.

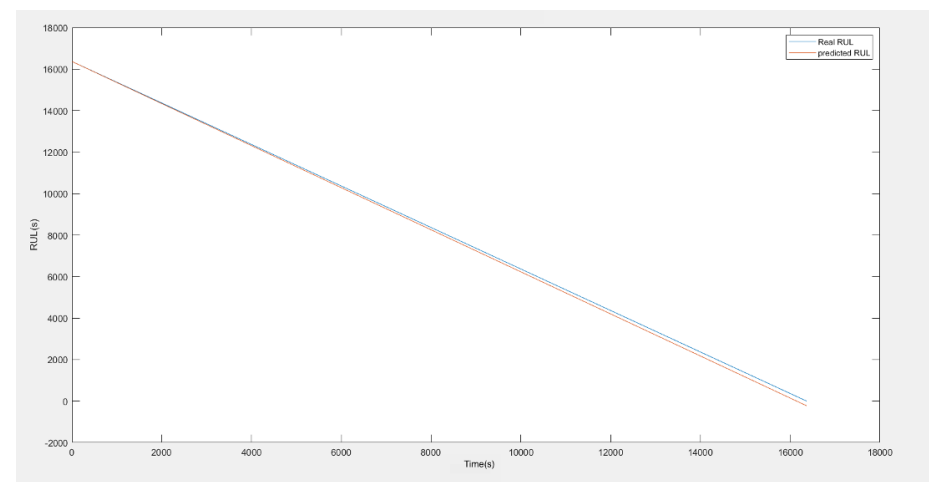

Fig. 2. RUL prediction as a function of time in seconds

To conclude, a method in the data-driven framework was proposed to estimate the RUL of a system. The method was applied to the data of PRONOSTIA with different results. The results show a better superposition to the real data than the results of [5].

\section{References}

1. R. Gouriveau, K. Medjaher and N. Zerhouni, From Prognostics and Health Systems Management to Predictive Maintenance 1: Monitoring and Prognostics. John Wiley \& Sons (2016).

2. B. Chebel-Morello, J.-M Nicod and C. Varnier. From Prognostics and Health Systems Management to Predictive Maintenance 2: Knowledge, Traceability and Decision. John Wiley \& Sons (2017).

3. P. Nectoux, R. Gouriveau, K. Medjaher, E. Ramasso, B. Chebel-Morello, N. Zerhouni, and C. Varnier. PRONOSTIA: An experimental platform for bearings accelerated degradation tests. In IEEE International Conference on Prognostics and Health Management, PHM'12, pp. 1-8. IEEE, (2012).

4. D. Galar and U. Kumar. EMaintenance: Essential Electronic Tools for Efficiency. Academic Press (2015).

5. K. Medjaher, N. Zerhouni, and J. Baklouti. Data-driven prognostics based on health indicator construction: Application to PRONOSTIA's data. In Control Conference (ECC), 2013 European, pp. 1451-1456. IEEE, (2013) 